\title{
Application Value of Rapid Predictive Model for Readmission Risk in Patients after CABG
}

\author{
Tiao Lv, Undergraduate, ${ }^{1}$ Yinghong Zhang, MD, PhD, ${ }^{1}$ Wen Zhang, $\mathrm{MD},{ }^{2}$ Liu Hu, MD,${ }^{2}$ \\ Guozhen Liu, Undergraduate, ${ }^{1}$ Hong Cheng, MD, PhD, ${ }^{1}$ Jing Huang, $\mathrm{MD}^{3}$ \\ ${ }^{1}$ School of Medicine, Wuhan University of Science and Technology, Wuhan, China; ${ }^{2}$ Department of Cardiology, Wuhan Asian \\ Heart Hospital, Wuhan, China; ${ }^{3}$ Wuhan Puren Hospital, Wuhan, Hubei, China
}

\section{ABSTRACT}

Objective: To explore the value of a rapid risk predictive model for the readmission of patients after CABG in China.

Methods: The rapid predictive model of readmission risk was translated into Chinese, and then validated with data from 758 patients who underwent CABG in Wuhan Asian Heart Hospital from January 2018 to June 2019. The discrimination was tested by area under the ROC curve (AUC), and the calibration was tested by Hosmer-Lemeshow test.

Results: The rapid risk predictive model for readmission showed good discrimination and calibration in Chinese CABG patients (The area under ROC curve c-statistic: 0.704, 95\% CI: 0.614-0.794; Hosmer-Lemeshow test: $P=.955$ ).

Conclusion: The rapid readmission risk predictive model can be used in Chinese CABG patients soon after admission.

\section{INTRODUCTION}

Coronary artery bypass grafting (CABG) surgery is internationally recognized as the most effective treatment for coronary heart disease (CHD); however, the readmission rate within 30 days after CABG reaches almost $15 \%$ and the annual cost of medical insurance is above $\$ 151$ million, which not only increases the burden on patients, but also adds higher expenditure to the public [Zywot 2017]. Compared with western countries [Zywot 2017; Benuzillo 2018; Rosenblum 2019; Khoury 2019], current research on coronary artery bypass grafting (CABG) in China has typically focused on postoperative complications such as pulmonary infection, vascular bridge disease, atrial fibrillation, and relative risk factors [Li 2017; Xi 2018; Fan 2019]. There has been far less research conducted on the model for prediction

Received Fune 7, 2020; received in revised form Fuly 21, 2020; accepted Fuly 22, 2020.

This work was supported by foint Foundation of Hubei Provincial Health Commission (W72019H345); and by College Student Innovation Fund of Wuban University of Science and Technology (18ZRA178).

Correspondence: Yinghong Zhang, School of Medicine, Wuban University of Science and Technology, Wuban, Hubei, China, 430065; (e-mail: zhangyinghong@wust.edu.cn).

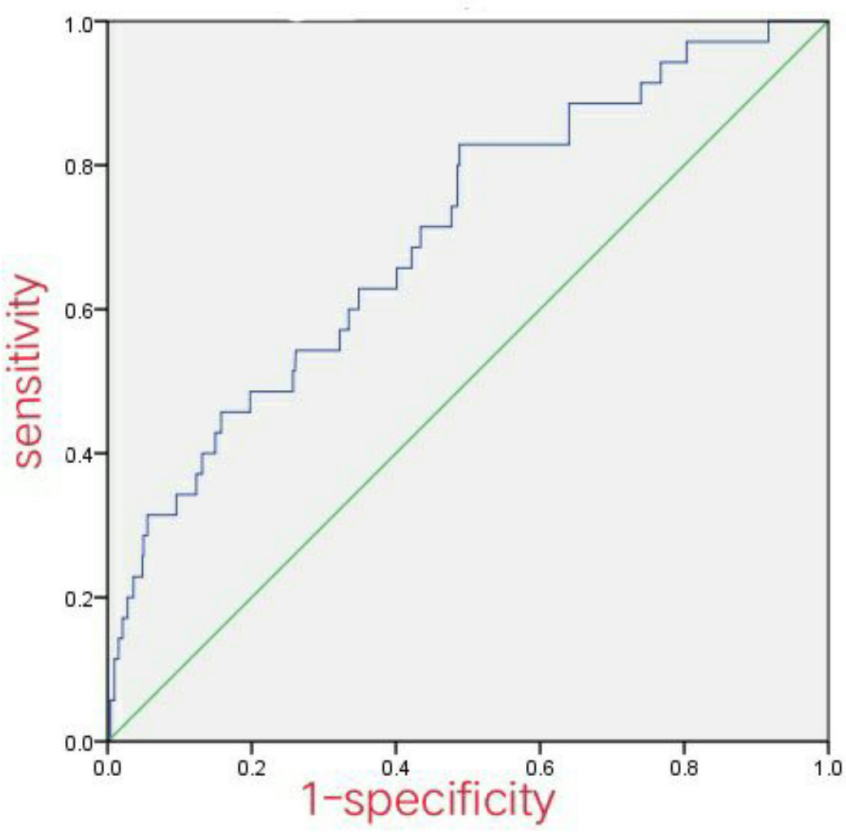

Figure 1. Area under the ROC curve (AUC) plots for predictive model fitted on the validation samples. ROC indicates receiver operating characteristic.

Table 1. Multivariate Predictors of 30-Day Readmission for CABG Patients and Formula for Calculating Readmission Risk[Benuzillo 2018]

\begin{tabular}{ll}
\hline Risk Factor & Estimate \\
\hline Age, $y$ & 0.027 \\
Albumin, $\mathrm{mg} / \mathrm{mL}$ & -0.373 \\
Prior heart failure & 0.436 \\
History of diabetes & 0.433 \\
Previous myocardial infarction & 0.366 \\
\hline & \\
Multivariable predictors of 30-day readmission and formula for calculating \\
the risk of readmission: logit $(P)=\ln (P / 1-P)=-3.28+0.027$ (age) +0.436 \\
(prior heart failure) -0.373 (total albumin) +0.366 (previous myocardial \\
infarction) +0.433 (history of diabetes).
\end{tabular}


Table 2. Comparison of Readmission and Non-Readmission of CABG Patients within 30 Days

\begin{tabular}{|c|c|c|c|c|c|}
\hline Age, y & $62(55-68)$ & $63(56-70)$ & $62(55-67)$ & $-1.370 *$ & .171 \\
\hline Albumin $(\mathrm{mg} / \mathrm{mL})$ & $38.85(36.5-41.325)$ & $37(33.7-40.8)$ & $38.9(36.6-41.4)$ & $-2.634 *$ & .008 \\
\hline Previous myocardial infarction & 246 & $19(54.3 \%)$ & $227(31.4 \%)$ & $7.978 \dagger$ & .005 \\
\hline
\end{tabular}

Data reported as median and quartile or percentage.

*indicates $Z$ value; $\dagger$ indicates $\chi^{2}$ value
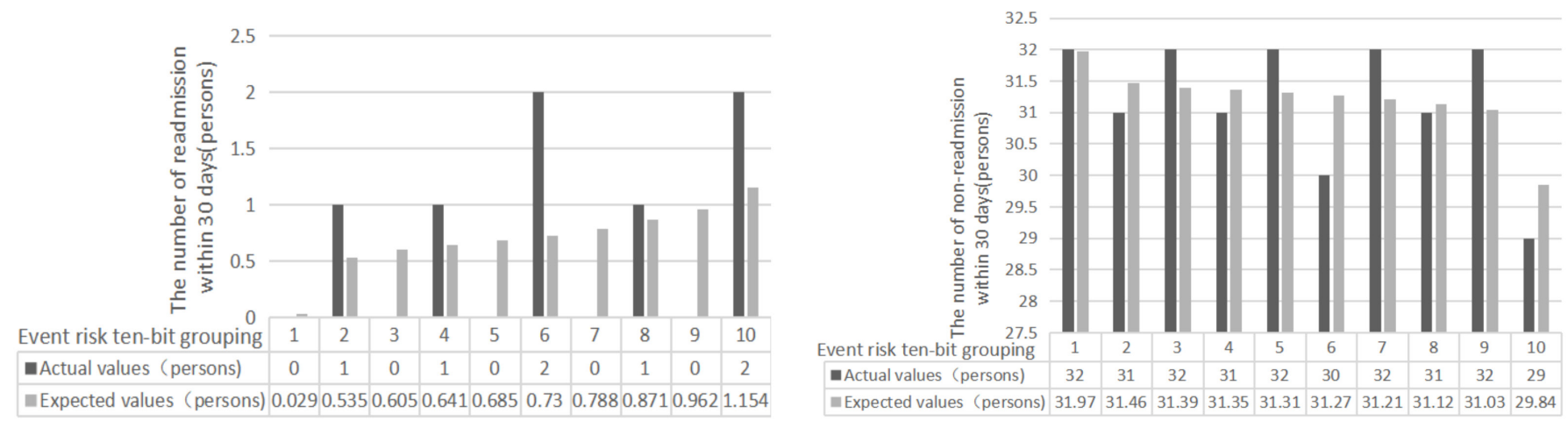

Figure 2. Calibration chart of readmission and non-readmission within 30 days in the medium-risk group.

of readmission 30 days post CABG surgery. On the other hand, the main interventions to reduce the risk of postoperative readmission in CABG patients are postoperative care and regular follow-up for all patients [Case 2020; Hammond 2015; Sood 2015], lacking different interventions for different populations at risk. A predictive model can be helpful in evaluating patients at high risk of readmission, and developing individualized interventions to reduce readmission rate [Benuzillo 2018]. At the same time, some research suggests that CABG risk of readmission is related to sex and race [Shah 2019; Deo 2018; Tseng 2018; Cho 2019]. In order to reduce demographic and geographical constraints, we introduced the rapid risk prediction model for readmission. The purpose of this study was to sinicize and validate the rapid risk prediction model for readmission, and explore its application value in Chinese patients with CABG.

\section{METHODS}

Our study was approved by Medical Ethics Committee of Wuhan University of Science and Technology (202042) before data collection. All records of patients who had undergone CABG from January 2018 to June 2019 in Wuhan Asian Heart Hospital were collected. Of 758 patients, 508 were male and 250 were female. Inclusion criteria: age $\geq 18$ years; patients underwent $C A B G$ surgery and were readmitted within 30 days. Exclusion criteria: suffering from organic diseases that may lead to mental disorders; patients with incomplete data.

Our research team was composed of 1 professor, 2 cardiology experts, 2 English experts, and 2 undergraduates. The rapid predictive model for the risk of postoperative readmission of CABG patients comprised five risk factors, including age, heart failure, total albumin, previous history of acute myocardial infarction, and diabetes [Benuzillo 2018] (Table 1). It was translated into a Chinese version to form the first draft; 2 English experts then translated the formula and compared it with the English version. The inconsistencies were discussed. After repeated comparison, the agreement was reached and the Chinese rapid predictive model for the risk of postoperative readmission in CABG patients was formed.

\section{Statistical Analysis}

Data were analyzed using SPSS 24.0 software (IBM, Armonk, NY, USA). Data did not conform to normal distribution after inspected; Mann-Whitney $U$ test and Pearson chi-square test were then used. The area under the ROC curve (AUC) was used to test its discrimination, and the calibration was tested with Hosmer-Lemeshow test. C statistic is one of the important indicators to describe the ability to discriminate in generalized linear models [Jia 2019]. The closer the $\mathrm{C}$ statistic is to 1 , the higher the discrimination ability 
Table 3. Comparison of Actual and Expected Values for CABG Patients with 30-Day Readmission

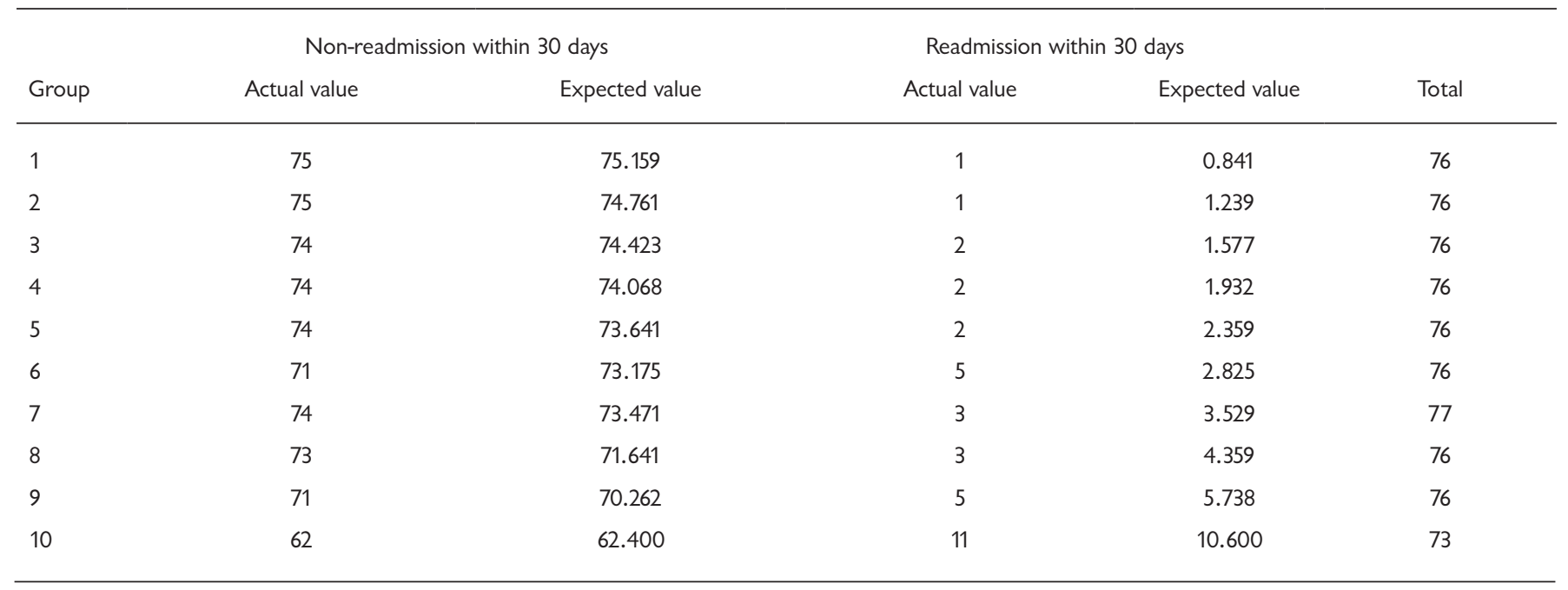
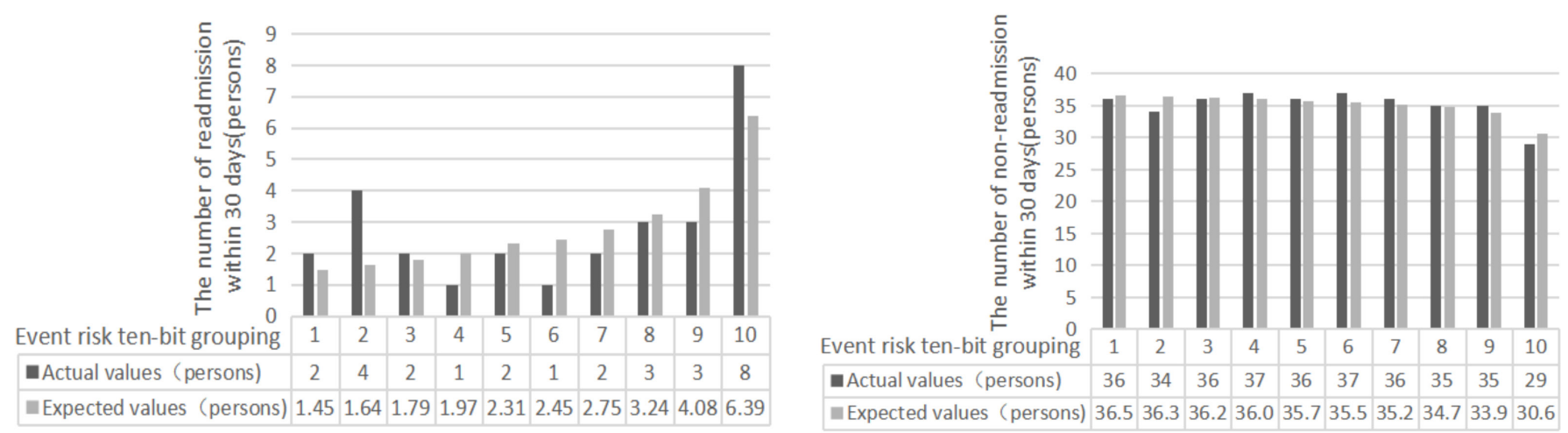

Figure 3. Calibration chart of readmission and non-readmission within 30 days in the high-risk group.

[Alba 2017]. Calibration degree is one of the best characteristics reflecting the prediction efficiency of the prediction model. Hosmer-Lemeshow goodness of fit test is generally used, and $P>.05$ indicates good calibration degree [Jia 2019].

\section{RESULTS}

\section{Baseline Characteristics}

Among 758 CABG patients, 35 were readmitted within 30 days after surgery $(4.62 \%)$. The differences of age and preoperative heart failure were not statistically significant $(P>.05)$. Significant predictors of 30-day readmission included albumin level, diabetes history, and previous myocardial infarction history $(P<.05)$. Lab values and preoperative medications are shown in Table 2.

\section{Validation of the Rapid Risk Predictive Model}

The area under the curve c-statistic obtained from the validation cohort was 0.704 (95\% CI: 0.614-0.794), which indicated that the model had good discrimination, as shown in Figure 1. According to the Hosmer-Lemeshow goodness of fit test $(P=.955)$, the model was shown to calibrate well (Table 3).

\section{DISCUSSION}

\section{Advantages of the Rapid Predictive Model}

According to the latest research, there are about 400,000 CABG cases in the United States each year, and about 40,000 per year in China [Wu 2017]. Many risk prediction models of cardiac surgery have been developed to reduce mortality [Ding 2016]. Research also suggested that, from 2004 to 2013, the mortality and complication rate of CABG patients decreased significantly [Hu 2019]. In China, there were many studies focused on CABG risk scoring model to decrease mortality [Ding 2016; Lin 2019]; however, there was less focus on research to develop models for reducing readmission.

Compared with other models, the rapid predictive model has advantages. The biggest advantage is that it can be used easily after patients' admission because it includes only five 
Table 4. Prediction Results and Grouping of the Rapid Assessment Model for Postoperative Readmission Risk of CABG Patients

\begin{tabular}{lccc}
\hline Group & Readmission rate, $\%$ & Predictive probability & $\begin{array}{c}\text { Number of cases } \\
\mathrm{n}(\%)\end{array}$ \\
\hline Low-risk group & $0 \leq$ Predictive probability<0.0130347 & $60(7.9)$ & 0 \\
Medium-risk group & $0.0130347 \leq$ Predictive probability<0.0331926 & $319(42.1)$ & $3.19 \%$ \\
High-risk group & $0.0331926 \leq$ Predictive probability $\leq 1$ & $379(50.0)$ & $7.39 \%$ \\
\hline
\end{tabular}

variables [Benuzillo 2018], while some models, such as the Medicare and Medicaid service center models, have 25 risk factors [Suter 2014]. From admission, patients with high risk of readmission will be given more incentive therapy and nursing, which can help reduce readmission within 30 days. Another advantage is that its application value can be evaluated among different populations. There are some models that include ethnic factors [Shah 2019; Deo 2018], which will limit their use worldwide. In contrast, the rapid predictive model does not comprise the ethnic factor, so it can be well adopted and validated.

\section{Application Value of Rapid Predictive Model in China}

In this study, the area under the ROC curve of the rapid predictive model for postoperative readmission risk of CABG patients was 0.704 , and the H-L test $P=.955>.05$. From the diagnostic test, the diagnostic value is low when the area under the ROC curve is between 0.5 and 0.7 , medium when it is between 0.7 and 0.9 , and high when it is above 0.9 [Song 2006]. Therefore, the rapid readmission risk assessment model has a high value for its good discrimination and calibration. According to the predicted risk probability, the patients were divided into three groups: low risk, medium risk, and high risk. The two risk thresholds for grouping were defined as the highest probability of maintaining a sensitivity of $100 \%$ and $80 \%$ [Zhen 2020], 0.0130347 and 0.0331926 , respectively. Of 758 patients, $60(7.9 \%), 319(42.1 \%)$, and 379 $(50.0 \%)$ were in the low-risk, medium-risk, and high-risk groups, respectively. The readmission rate within 30 days in each group was $0,2.19 \%$, and $7.39 \%$, respectively, as shown in Table 4. The calibration degree of each group was tested separately, and the low-risk group was not undergone the calibration degree test because the number of patients readmitted within 30 days was 0 , and there was no positive event. The expected and measured values of readmission and nonreadmission within 30 days for each group were compared. In the medium-risk group, the maximum difference between the expected and measured values of readmission and nonreadmission within 30 days was 1.27 , the minimum difference was 0.029 , as shown in Figure 2. In the high-risk group, the maximum difference between the expected and measured values of readmission and non-readmission within 30 days was 2.359 , the minimum difference was 0.209 , as shown in Figure 3 . The difference between the expected value and the measured value of the two groups was small and close to the actual situation. According to the risk grouping, the readmission rate of the high-risk group was higher than that of the medium-risk group and the low-risk group, which was consistent with the reality. The 30 -day readmission rate of the lowrisk group was 0 . The difference between the expected value and the measured value obtained by each group according to the test of calibration degree was small, which was in line with the actual situation. It indicates that the grouping results based on the highest probabilities of sensitivity of $100 \%$ and $80 \%$ as two critical points show consistency with the actual situation, which further indicates that the calibration degree of this model is good.

However, further investigation is required in the future due to (1) the insufficiency of management of patients' electronic medical record in Chinese hospitals, some data may be missed. For example, a patient may have been admitted to another hospital within 30 days after CABG surgery, or died of an unexpected illness; and (2) the model developed from data of Chinese CABG patients may improve the predictive value of the model in China.

\section{Conclusion}

This study shows that the rapid predictive model for readmission risk among CABG patients has good discrimination and calibration, which has a high application value in Chinese patients who undergo CABG. It can be useful for medical personnel to predict the risk of postoperative readmission of CABG patients soon after admission, and to take effective measures in a timely way to reduce the readmission rate within 30 days after surgery.

\section{REFERENCES}

Alba AC, Agoritsas T, Walsh M, et al. 2017. Discrimination and calibration of clinical prediction models: users' guides to the medical literature. JAMA 318:1377-84.

Benuzillo J, Caine W, Evans SR, et al. 2018. Predicting readmission risk shortly after admission for CABG surgery. J Card Surg 33:163-70.

Case R, George J, Li Q, et al. 2020. Unplanned 30-day readmission after coronary artery bypass in patients with acute myocardial infarction. Cardiovasc Revasc Med 21:518-21.

Cho BC, DeMario VM, Grant MC, et al. 2019. Discharge hemoglobin level and 30-day readmission rates after coronary artery bypass surgery. Anesthesia \& Analgesia 128:342-8.

Deo SV, Raza S, Altarabsheh SE, et al. 2018. Risk calculator to predict 30-day readmission after coronary artery bypass: a strategic decision support tool. Heart Lung Circulation 28:1896-903. 
Ding Y, Qi X, Zhao Y. 2016. Research progress of risk prediction models for patients undergoing coronary artery bypass grafting (in Chinese). Chin J Clin Thorac Cardiovasc Surg 23:1177-82.

Fan G, Zhang Y, Chen T, et al. 2019. Risk factors of pulmonary infection after coronary artery bypass grafting (in Chinese). Chinese Circ J 34:139-43.

Hammond DA, Smotherman C, Jankowski CA, et al. 2015. Short-course of ranolazine prevents postoperative atrial fibrillation following coronary artery bypass grafting and valve surgeries. Clinical Research in Cardiol 104:410-7.

Hu S. 2019. Development of cardiac surgery in China since the founding of the People's Republic of China (in Chinese). Chinese Circ J 34:937-43.

Jia Y, Zhou J, Chen Y, et al. 2019. Study on comprehensive evaluation system of clinical prediction model (in Chinese). Chinese J Health Stat 36:728-34.

Khoury H, Sanaiha Y, Rudasill SE, et al. 2019. Readmissions following isolated coronary artery bypass graft surgery in the United States (from the Nationwide Readmissions Database 2010 to 2014). Am J Cardiol 124:205-10.

Li Li, Zhao Qiang, Gai Luyue, et al. 2017. Outcome and influencing factor analysis for graft vessels in patients after coronary artery bypass grafting (in Chinese). Chinese Circ J 32:128-31.

Lin H, Hou J, Tang H, et al. 2019. A novel risk stratification system for predicting in-hospital mortality following coronary artery bypass grafting surgery with heart failure (in Chinese). Chinese Circ J 34:444-8.

Rosenblum JM, Lovasik BP, Hunting JC, et al. 2019. Predicted risk of mortality score predicts 30-day readmission after coronary artery bypass grafting. Gen Thorac Cardiovasc Surg 67:661-8.

Shah R M, Zhang Q, Chatterjee S, et al. 2019. Incidence, cost, and risk factors for readmission after coronary artery bypass grafting. Annals of Thorac Surg 107:1782-9.
Song Hualing, He Jia, Huang Pinxian, et al. 2006. Application of parametric method and non-parametric method in estimation of area under ROC curve (in Chinese). Acad J Second Military Med University 27:726-8.

Sood A, Abdollah F, Sammon JD, et al. 2015. The effect of body mass index on perioperative outcomes after major surgery: results from the National Surgical Quality Improvement Program (ACS-NSQIP) 20052011. World J Surg 39:2376-85.

Suter L, Wang C, Araas M, et al. 2014. Hospital 30-day readmission following coronary artery bypass graft surgery: updated measure methodology report 2014. http://www.cms.http://www.cms.gov/Medicare/ Quality-InitiativesPatient-Assessment-Instruments/HospitalQualityInits/Downloads/Coronary-Artery-Bypass-Graft-CABG-Readmission. zip [Accessed October 17, 2017].

Tseng HS, Chao ZH, Huang SK, et al. 2018. Utilization of emergency and hospitalization care after coronary artery bypass surgery for patients with ischemic heart disease. International Heart J 59:941-50.

Wu M, Zhou Z. 2017. Coronary artery bypass grafting (in Chinese). Chin J Health Care Med 19:187-91.

Xi L, Wang Z, Liu Z, et al. 2018. Risk predictors of new-onset atrial fibrillation after coronary artery bypass grafting (in Chinese). Chinese Circulation J 33:969-72.

Zhen L, Chuanhai G, Yujie H, et al. 2020. A clinical model predicting the risk of esophageal high-grade lesions in opportunistic screening: a multicenter real-world study in China. Gastrointestinal Endoscopy 91:1253-60.

Zywot A, Lau CSM, Glass N, et al. 2017. Preoperative scale to determine all-cause readmission after coronary artery bypass operations. Ann Thorac Surg 105:1086-93. 